\title{
Large-Format Distributed Read-Out Imaging Devices for X-Ray Imaging Spectroscopy
}

\author{
Roland den Hartog ${ }^{1}$, A. Kozorezov ${ }^{2}$, D. Martin ${ }^{1}$, G. Brammertz', P. Verhoeve' , A. Peacock, F. \\ Scholze$^{3}$, D. J. Goldie ${ }^{4}$
}

'Space Science Dept. of the European Space Agency, ESTEC, PO Box 299,2200 AG, Noordwijk, Netherlands

${ }^{2}$ Department of Physics, Lancaster University, Lancaster LA1 4 YB, U.K. ${ }^{3}$ Physikalisch-Technische Bundesanstalt (PTB), Abbestr. 2-12, D-10587 Berlin, Germany ${ }^{4}$ Oxford Instruments Scientific Research Division, Newton House,Cambridge Business Park, CB4 4WY, U.K.

ESLAB 2001/060

\section{0}

To be published in the Proceedings of the $9^{\text {th }}$ International Workshop on "Low Temperature Detectors", 23-27 July 2001, Madison, WI, USA

CERN LIBRARIES, GENEVA 


\title{
Large-Format Distributed Read-Out Imaging Devices for X-Ray Imaging Spectroscopy
}

\author{
Roland den Hartog ${ }^{1}$, A. Kozorezov², D. Martin', G. Brammertz', P. Verhoeve', \\ A. Peacock ${ }^{1}$, F. Scholze ${ }^{3}$, D.J Goldie ${ }^{4}$ \\ ') Space Science Dept. of the European Space Agency, ESTEC, PO BOx 299, 2200 AG, Noordwijk, Netherlands \\ -) Department of Physics, Lancaster University, Lancaster LAI AYB. U.K. \\ 3) Physikalisch-Techinische Bundesanstalt (PTB), Abbestr. 2-12, D-10587 Berlin, Germany \\ $\left.{ }^{4}\right)$ Oxford Instruments Scientific Research Division, Newton House, Cambridge Business Park, CB4 4WY, U.K.
}

\begin{abstract}
We present an experimental study of the performance of Distributed Read-Out Imaging Devices (DROIDs), based on two Ta/Al-based superconducting tunnel junctions (STJs) placed on either side of a Ta absorber strip. We focus our discussion on the prospects of building large-format photon-counting imaging spectrometers for applications at optical. UV and soft X-ray energies. Tunnel-limited spectroscopical resolutions have already been demonstrated for optical photons. With a $20 \times 100 \mu \mathrm{m}^{2}$ absorber we have measured an intrinsic energy resolution of $2 \mathrm{eV}$ FWHM for $500 \mathrm{eV}$ photons. This demonstrates that at soft X-ray energies resolutions close to the tunnel limit are also feasible for this type of detectors. A detailed analysis of pulse-shapes with numerical models allows us to assess the main parameters that determine the performance of these detectors. Extrapolation of these models indicates that it is possible to extend the length of the absorber to $1.5 \mathrm{~mm}$, without a serious degradation of the detector's performance.
\end{abstract}

The next generation of astronomical X-ray missions places challenging demands on the instrumentation. The foreseen $X$-ray detectors on these missions should combine high spectroscopic resolution with imaging capability in large-size pixels at high photon counting rates. Taking ESA's X-ray mission XEUS as an example [1, 2], the requirements for the Xray imaging spectroscopy detectors, NFI-1 and 2, include a pixel size $\geq 100 \mu \mathrm{m}$ with a total detector area of $7 \times 7 \mathrm{~mm}^{2}$, an energy resolution of $0.1 \%$ of the photon energy in energy ranges
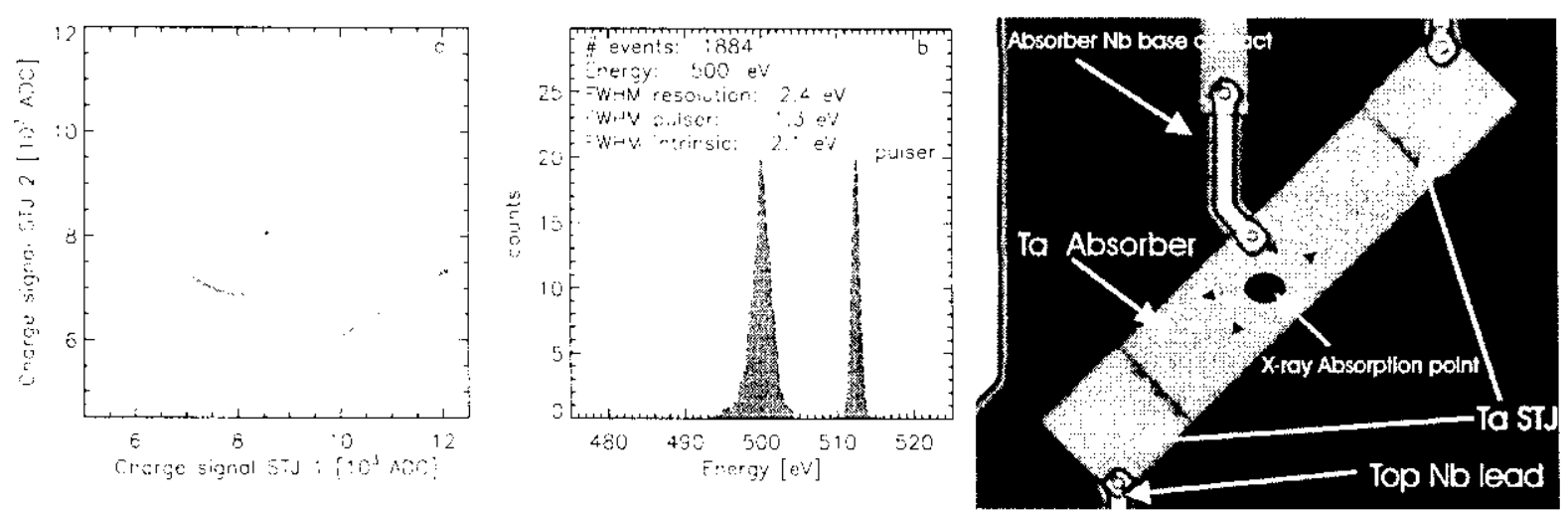

FIGURE 1. $a$. Charge signals of the two STJs of a $20 \times 100 \mu \mathrm{m}^{2}$ DROID plotted against each other. The energy was $500 \mathrm{eV}$. Note that the DROID was not completely illuminated due to the settings of the synchrotron beam. $b$. The corresponding spectrum, obtained by taking events from the central $-30 \%$ of the absorber area, in a $180 \mathrm{~s}$. time window. $c$. A top view Nomarski microscope image of this DROID. 
from $0.1-2$ and $0.5-10 \mathrm{keV}$ respectively, and $\leq 5 \mu \mathrm{s}$ time resolution. Cryogenic detectors, such as microcalorimeters and STJs, appear the most promising technology, because they offer, compared to CCDs, a superior energy resolution, a broad energy range, and the possibility of single photon counting up to rates of several tens of $\mathrm{kHz}$. However, the necessity to individually bias and read-out each device implies at least one electrical connection per pixel, which poses a serious challenge to the development of the equivalent of a large-format imaging array $[3,4]$.

A possible solution is offered by DROIDs, in which photons are absorbed either in a single crystal of large dimensions [5] or a smaller poly-crystalline layer [6-8], and subsequently detected by STJs located at the edges of the absorber (see Fig. 1c). Compared to an array, the DROID geometry provides a large sensitive area with a considerable reduction in the number of read-out connections. By time-coincident event measurement it is possible to reconstruct both the absorption position and the energy of the incoming photons. The second main advantage of this type of detector is therefore that they allow a correction for the dependence of the charge signal on the absorption position, which is the main source of the degradation of energy resolution in single STJs $[9,10]$. As a result, energy resolutions close to the statistical limit are feasible.

This is demonstrated in Fig. 1b, where we show the spectrum for $500 \mathrm{eV}$ photons, taken with a $20 \times 100 \times 0.1 \mu^{3}$ Ta DROID, with Ta/Al STJs, at the PTB SX-700 beamline at the Bessy II synchrotron facility in Berlin. The intrinsic energy resolution $2.1 \mathrm{eV}$ is close to the computed statistical limit of $2.0 \mathrm{eV}$. Fig. 2b shows that it is still a challenge to routinely operate this device close to the tunnel limit. The main limitation is set by external noise sources, presumably related to the stability of the bias circuit. This can be concluded from Fig. $2 b$, in which the difference in resolution between two subsets, each containing half the number of events, by far exceeds the formal statistical errors. Fig. 1 was obtained during a brief period of, apparently, very low noise. We estimate that an improvement of the bias stability by at least a factor 10 is required. Finally, we note that Fig. $2 \mathrm{c}$ demonstrates that for
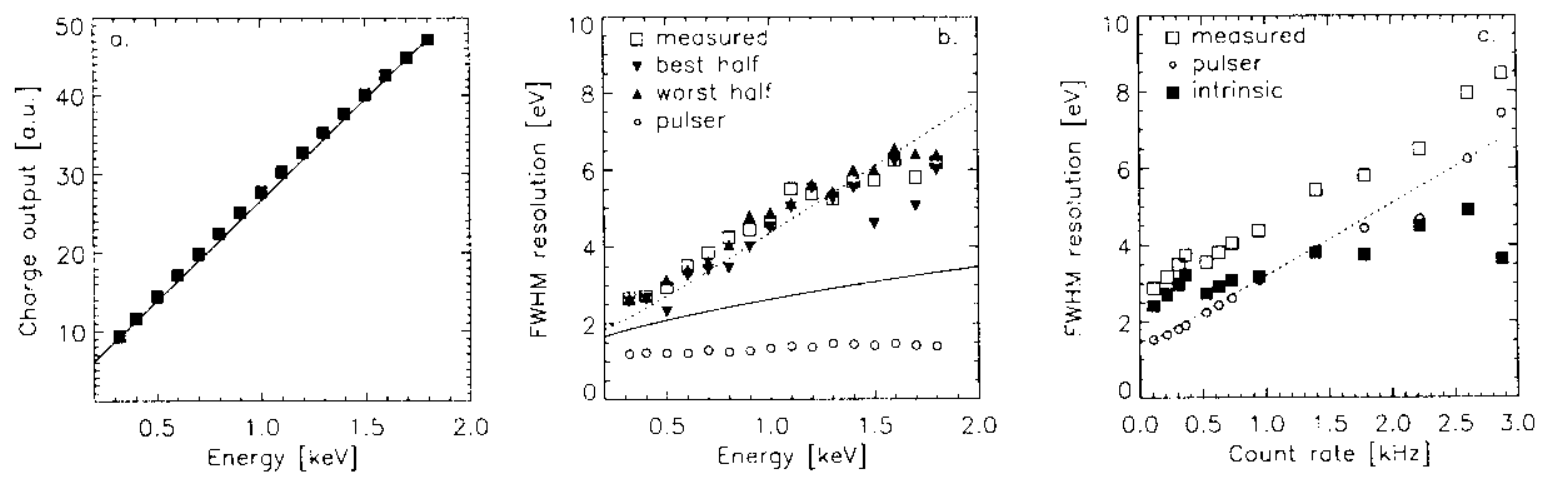

FIGURE 2. $a$. Charge output as a function of photon energy for the $20 \times 100 \mu \mathrm{m}^{2}$ DROID, showing a high degree of linearity. The solid line indicates exact linearity. The residual non-linearity is due to qp selfrecombination. $b$. Energy resolution as a function of photon energy. Squares indicate measured resolutions, circles indicate the electronic noise as measured with the pulser, up and down triangles the two half subsets of the data with resp. the worse and the better energy resolution. The solid line indicates the statistical limit of the energy resolution, corresponding to a sum of Fano and tunnel factor $F+G=1.28$, while the dotted line indicates the best fitting curve including a contribution for the bias instability. $c$. Energy resolution for $500 \mathrm{eV}$ photons as a function of countrate. The dotted line indicates the average pulser width. The shaping time for these measurements was $10 \mu \mathrm{s}$, so the pile-up is mainly due to the pulse duration. 

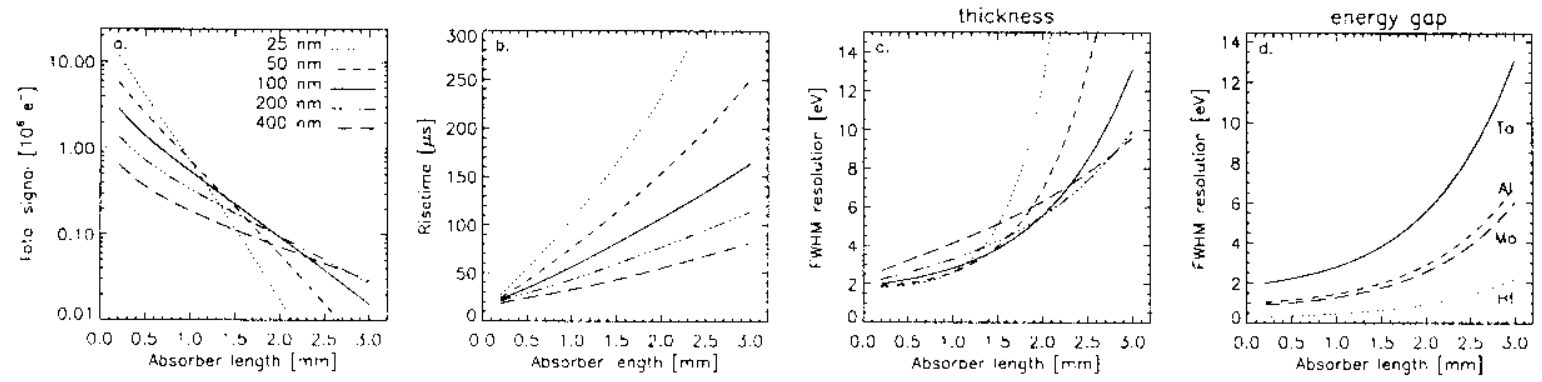

FIGURE 3. Simulation of measurements of $500 \mathrm{eV}$ radiation with large-format DROIDs. The models assume two $1 \mu \mathrm{m}$ wide ground connections on either side of the $30 \mu \mathrm{m}$ wide absorber. The STJs are $30 \times 30 \mu \mathrm{m}^{2} . a$. The total charge output as a function of absorber length $L$, for various thickness values $d$ of the Ta absorber. Note that we assume that the diffusion constant in the absorber scales as $D \propto R R R \propto d . b$. Pulse risetimes (time in which l/e of the total charge is collected) for photon absorption in the center of the Ta absorber, as a function of $L$. $c$. FWHM energy resolution vs. $L$ for various thicknesses $d$ of the Ta absorber. $d$. FWHM energy resolution vs. $L$ for different absorber materials. Here we assume $d=100 \mathrm{~nm}$.

count rates up to at least $1 \mathrm{kHz}$ there is no significant degradation of the energy resolution. The disadvantages of the DROID approach are the relatively lower charge output, because the quasiparticle charge carriers (qps) are spread out over a larger volume, and an increase in pulse duration times, due to longer qp diffusion times from the photon absorption site to the detectors. Both effects have a negative influence on the energy resolution. At lower charge outputs the statistics are less favorable, resulting in a lower signal-to-noise ratio, and for longer pulse durations, the probability for pile-up of events increases. As these problems tend to increase for larger absorber sizes, it is important to ensure that they do not prohibit a succesful large-format detector.

To this end we have developed an analytic model which describes the shapes of the two pulses emerging from a DROID, $I_{1,2}(t)$, in terms of the DROID geometry (absorber length $L$, thickness $d$ and width) and a set of physical parameters: the qp diffusion constant in the absorber $D$, the tunnel time of the qps in the STJs $\tau_{\text {tun }}$, and the qp loss times in the absorber $\tau_{\mathrm{abs}}$, the STJ top electrodes $\tau_{\text {top }}$, and the base contact lead to the absorber, $\tau_{\text {lead. }}$. This model was derived from the theory described in Ref. [9]. The values for the model parameters were obtained by fitting 550 pairs of pulse shapes from a $50 \times 400 \times 0.1 \mu \mathrm{m}^{3}$ DROID, sampled with a digital oscilloscope and selected from 11 different photon-absorption positions on the absorber. We used the longest absorber in our collection to make the extrapolation to larger sizes as faithful as possible. The fitted parameter values are: $D=17 \mathrm{~cm}^{2} \mathrm{~s}^{-1}, \tau_{\text {tun }}=4.3 \mu \mathrm{s}$, $\tau_{\mathrm{abs}}=52 \mu \mathrm{s}, \tau_{\mathrm{top}}=2.1 \mu \mathrm{s}$ and $\tau_{\text {lead }}=5.2 \mu \mathrm{s}$.

We apply the model to a DROID lay-out which was improved with respect to the one shown in Fig. 1c: the $3 \mu \mathrm{m}$ wide central lead, responsible for most of the curvature in the relation between $Q_{1}$ and $Q_{2}$ in Fig. 1a, is replaced by two $1 \mu \mathrm{m}$ wide leads on the far ends of the absorber. As a result, total charge output and energy resolution are (almost) independent of photon-absorption position, and the models have to be evaluated only at one position (in this case the center of the absorber). Note that charge output calculations refer to the pure STJ output and have not been convolved with any electronic transfer function. For the energy resolution, only the contributions from Fano and tunnel noise are taken into account. Cancellation noise $[11,12]$ is in our case negligible, because of the high $V_{\text {bias }}$ at which we can operate our STJs. Fig. 3a illustrates the fact that making the absorber thinner, reduces its 
volume and increases the qp density. For small absorber sizes this promotes the tunnel rate, and hence the charge output. For larger absorber sizes, a thinner absorber implies slower qp diffusion, as $D \propto R R R \propto d$, which means that more qps get lost before they tunnel, and thus reduces the charge output. To avoid pile-up thicker absorbers are better than thinner, as can be seen in Fig. $3 b$, because the pulse duration time scales roughly inversely proportional to the qp diffusion constant. A $400 \mathrm{~nm}$ thick, $1 \mathrm{~mm}$ long absorber has roughly the same pulse risetime as a $100 \mathrm{~nm}$ thick, $0.2 \mathrm{~mm}$ long absorber. Because the energy resolution is directly proportional to the signal-to-noise ratio and scales as $Q_{\text {tot }}{ }^{-1 / 2}$, it is lowest when the charge output is highest. For absorber lengths between 1.2 and $2 \mathrm{~mm}, 100 \mathrm{~nm}$ seems to be the optimal thickness. Finally, we show the effect of different materials, which is straightforward: energy resolution scales as $\Delta_{\mathrm{gap}}{ }^{1 / 2}$. Other material constants have not been taken into account. We implicitly assume that it is possible to deposit absorbers with the same diffusive properties as the present Ta absorbers. For $\mathrm{Al}$ and Mo, films with high residual resistance ratios (RRR) have already been deposited, while the Fermi velocity and mean-free path in these materials is higher than in Ta, implying that high values for $D$ are likely. $\mathrm{Al}$ is of course not a good choice because of its low X-ray stopping power, but it may be interesting for optical applications. For Hf, however, the deposition of a high-quality absorber is more challenging [13]. Moreover, the basic timescale for phonon-qp scattering processes, $\tau_{0}$, which scales as $\Delta_{\text {gap }}{ }^{-3}$, is estimated to be $217 \mu \mathrm{s}$ in Hf [14], implying that qps will hardly thermalise during their diffusion to the STJs, even in the large-format DROIDs. So, also in terms of material properties a trade-off has to be made.

In conclusion, a $200 \mathrm{~nm}$ thick, $1.25 \mathrm{~mm}$ long Mo absorber seems optimal in terms of size, energy resolution, pile-up and technical feasibility to meet the requirements for the XEUS NFI-1 instrument. Compared to the DROIDs tested in this study, its minimum energy resolution should be close to $1.5 \mathrm{eV}$ for $500 \mathrm{eV}$ photons, while the pulse risetime is only twice as long. In fact, pulses may turn out shorter, thus alleviating the pile-up problem, because in Mo both the Fermi velocity and the mean-free path are better than in Ta, and films with RRR values $>30$ (at $100 \mathrm{~nm}$ thickness) have already been produced in this material [15].

\section{REFERENCES}

[1] M. Arnaud, X. Barcons, et al., X-ray Evolving-Universe Spectroscopy - The XEUS Science Case, ESA-SP $1238(2000)$

[2] M. Bavdaz, A. Peacock, A.Parmar, M. Beijersbergen and J. Schiemann, Proc. of the $2^{\text {ndl }}$ European Symposium on the Utilisation of the International Space Station, ESA-SP 433, 621 (1999)

[3] D. Martin, P. Verhoeve, A. Peacock and D.J. Goldie, Proc. SPIE 4008, 328 (2000)

[4] P. Verhoeve, S. Kraft, N. Rando, A. Peacock et al., IEEE Trans. Appl. Supercond. 9, 3342 (1999)

[5] M.L. van den Berg, F.B. Kiewiet, M.P. Bruijn et al., IEEE Trans. Appl. Supercond. 9, 2951 (1999)

[6] H. Kraus, F. von Feilitzsch, J. Jochum, R.L. Mössbauer et al., Phys. Lett. B 231, 195 (1989)

[7] K. Segall, C.M. Wilson, L. Li, A.K. Davies et al., IEEE Trans. Appl. Supercond. 9, 3326 (1999)

[8] S. Friedrich, K. Segall, M.C. Gaidis, C.M. Wilson, D.E. Prober et al., Appl. Phys. Lett. 71, 3901 (1997)

[9] A. Kozorezov, J.K Wigmore, R. den Hartog, D. Martin, P. Verhoeve, A. Peacock, Phys. Rev. B (submitted)

[10] R. den Hartog, A. Kozorezov, J.K Wigmore, D. Martin, P. Verhoeve, A. Peacock, Phys. Rev. B (submitted)

[11] K. Segall, PhD thesis, Yale University, chapter 5 (1999)

[12] K. Segall, C.M. Wilson, L. Frunzio, L. Li, S. Friedrich et al., Appl. Phys. Lett. 76, 3398 (2000)

[13] S. Kraft, A. Peacock, M. Bavdaz, B. Castelletto, B. Collaudin, D. Perez et al., Proc. SPIE 3445, 226 (1998)

[14] A. Poelaert, PhD thesis, Twente University, chapter 6 (1999)

[15] A. Peacock, R. Venn, J. van der Biezen, int. comm. (1999) 